\title{
Intravascular ultrasound imaging in evaluation of aortic stiffness: A proof-of-concept study
}

\author{
Niya Boykova Mileva ${ }^{\circledR}$, Dobrin Iotkov Vassilev \\ Cardiology Clinic, “Alexandrovska” University Hospital, Medical University of Sofia, Bulgaria
}

\begin{abstract}
Background: Aortic stiffness is a well-known cardio-vascular risk factor. For years, different methods have been studied in the assessment of aortic elastic properties and large arterial stiffness for risk stratification. Herein is an assessment of the role of intravascular ultrasound (IVUS) imaging for the evaluation of aortic elastic properties.

Methods: Intravascular ultrasound imaging of the aorta was performed in 12 patients with transthoracic echocardiography (TTE) and computed tomography (CT) evidence for enlargement of the ascending aorta - diameter $\geq 40.0 \mathrm{~mm}$. Mechanical properties of the aorta were derived from the measured diameters and intra-aortic pressure. Paired samples T-test analyses were performed to determine differences between measurements derived by TTE, CT and IVUS.

Results: Mean values of the calculated elastic properties via IVUS of the ascending aorta were as follows: compliance $0.021 \pm 0.02$; strain $205 \pm 4.3$; aortic stiffness index $4.3 \pm 0.75$; elastic modulus $0.31 \pm 0.05$. On paired T-test analysis maximum ascending aortic diameter measured by CT aortography and IVUS did not differ significantly $(t=-0.19, p=0.985)$, but a significant difference between IVUS measurements and TTE derived diameters was found $(t=13.118, p=0.034)$. On average, IVUS diameters were $2.3 \mathrm{~mm}$ larger than the results acquired by TTE (95\% confidence interval: 14.21-17.13). Conclusions: Intravascular ultrasound examination of the ascending aorta provided larger diameters than the ones collected by means of TTE. However, IVUS measurements did not differ significantly from diameters derived by CT aortography. (Cardiol J 2023; 30, 2: 221-227)
\end{abstract}

Key words: aortic stress, intravascular ultrasound, aortic compliance

\section{Introduction}

Large elastic arteries in the human body have the ability to stretch in response to the ventricular contractions [1]. They act as an elastic buffering chamber enabling a relatively continuous peripheral blood flow during the cardiac cycle [2]. With aging, there is a response to the cumulative exposure to hemodynamic loading; lifestyle and cardiovascular (CV) risk factors in large arteries and especially the aorta are prone to abnormal stiffening [3]. Aortic stiffening plays central role in the pathophysiology of arterial hypertension, left ventricular remodeling, heart failure. Furthermore, it may have a major impact on CV health as it has been proved as an independent predictor of mortality as well as predictor of ischemic heart disease and stroke in the general population $[4,5]$. For years, different methods have been studied in the assessment of aortic stress and large arterial stiffness for risk stratification. The present study assesses the role of intravascular ultrasound (IVUS) imaging for the evaluation of aortic elastic properties.

Address for correspondence: Niya Mileva, MD, “Alexandrovska” University Hospital, Medical University, “St. George Sofiiski” Str. 1, 1431 Sofia, Bulgaria, tel: +35929230467, e-mail: nmileva91@gmail.com

Received: 28.03.2020 Accepted: 13.10.2020 Early publication date: 11.01.2021

This article is available in open access under Creative Common Attribution-Non-Commercial-No Derivatives 4.0 International (CC BY-NC-ND 4.0) license, allowing to download articles and share them with others as long as they credit the authors and the publisher, but without permission to change them in any way or use them commercially. 
Table 1. Maximal measurements of ascending aorta derived by transthoracic echocardiography (TTE), computed tomography (CT) aortography and intravascular ultrasound (IVUS).

\begin{tabular}{lccc}
\hline & $\begin{array}{c}\text { Maximal TTE } \\
\text { diameter }[\mathrm{mm}]\end{array}$ & $\begin{array}{c}\text { Maximal CT } \\
\text { diameter }[\mathrm{mm}]\end{array}$ & $\begin{array}{c}\text { Maximal IVUS } \\
\text { diameter [mm] }\end{array}$ \\
\hline Patient 1 & 43 & 46 & 48 \\
Patient 2 & 46 & 44 & 47 \\
Patient 3 & 48 & 49 & 51 \\
Patient 4 & 49 & 48 & 50 \\
Patient 5 & 46 & 50 & 50 \\
Patient 6 & 50 & 49 & 47 \\
Patient 7 & 47 & 46 & 46 \\
Patient 8 & 57 & 59 & 58 \\
Patient 9 & 39 & 41 & 43 \\
Patient 10 & 50 & 53 & 52 \\
Patient 11 & 52 & 60 & 60 \\
Patient 12 & 43 & 45 & 46 \\
Mean & 47.5 & 49.2 & 49.8 \\
\hline
\end{tabular}

\section{Methods}

\section{Patient selection}

- Subject $\geq 18$ years old;

- Evidence from transthoracic echocardiography (TTE) of enlargement of the ascending aorta diameter $\geq 40.0 \mathrm{~mm}$;

- Evidence from 64-slice multislice computed tomography (CT) angiography for enlargement of the ascending aorta - diameter $\geq 40.0 \mathrm{~mm}$.

\section{Procedures}

All subjects involved signed informed consent forms approved by the documented institution. The $20 \mathrm{MHz}$, Visions PV probe (Volcano Philips Corp, USA) through an $8.5 \mathrm{~F}$ femoral sheath was utilized. By virtue of manual pullback of the probe, the vessel was visualized through its whole length from the aortic root to the aorto-iliac bifurcation. Measurements of maximal systolic and diastolic cross-sectional area and diameters of the vessel were obtained at every $1 \mathrm{~mm}$. Simultaneous recording of intra-aortic systolic (Ps) and diastolic pressures (Pd) were also performed (Table 1 ).

The following definitions and calculations were used for the analysis [6, 7].

For the calculation of wall properties, it was assumed that the cross-section of an artery is circular.

- Diameter: $D=\sqrt{ }(A / \pi)$;

- Arterial compliance $(\mathrm{C})$ is the absolute change in area (or change in diameter $\mathrm{D}$ ) for a given pressure step $(\mathrm{P})$ at a fixed vessel length. The terms "compliance" and "elasticity" are often used interchangeably: $C=\Delta D / \Delta P$;

- Stress is defined as the force applied to a particular object or area. It can be applied in radial, circumferential, and longitudinal directions. Circumferential wall stress, defined by Laplace's law, is directly proportional to the vessel pressure and radius and is inversely proportional to its thickness: Stress $=F / A$;

- Strain is the resulting deformation (percentage change in length) of an object/material subjected to a stress force: Strain $=(D D-S D) / D D$;

- Stiffness was defined as the resistance offered by an elastic body to deformation. Aortic stiffness index: $\beta=\ln (S B P / D B P) /$ strain, where 'In' means natural logarithm;

- The elastic modulus (E), is the stress/strain ratio. Elastic modulus: $E(p)=(S B P-D B P) /$ strain;

- Distensibility was defined as the change in diameter/area/pressure step increase. It is the inverse of the elastic modulus (E): Aortic distensibility $=(2 \times$ strain $) /(S B P-D B P)$.

\section{Statistical analysis}

Statistical analysis was performed using IBM SPSS Statistics 23. Continuous variables were presented as mean \pm standard deviation. Categorical data were presented as numbers in percentages. Paired samples T-test analysis was performed to determine difference between measurements derived by TTE, CT and IVUS. If distribution was not normal (verified with the Kolmogorov-Smirnov test), the 


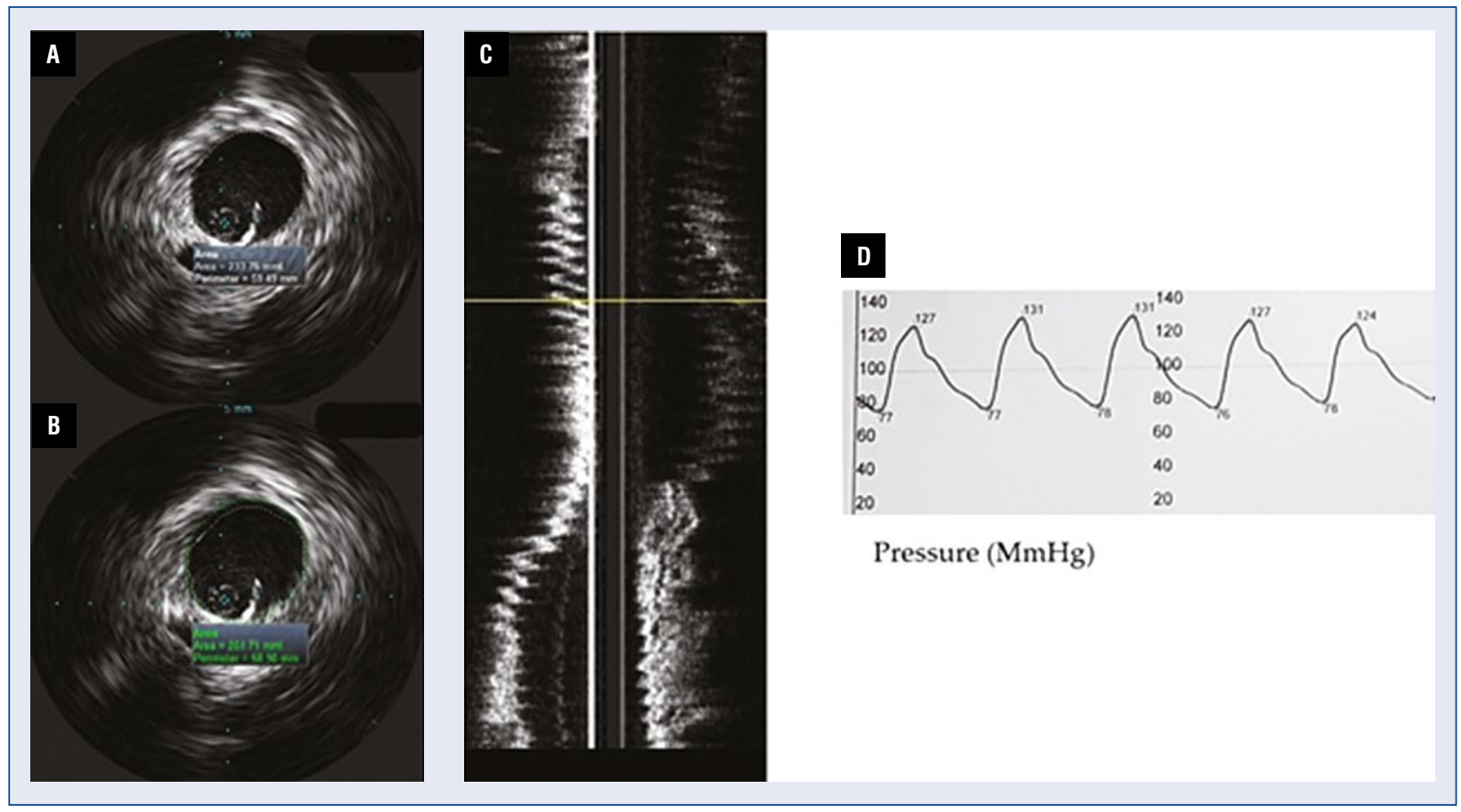

Figure 1. Evaluation of aortic mechanical properties; A. Diastolic cross-sectional lumen area of ascending aorta; B. Systolic cross-sectional lumen area of ascending aorta; C. Longitudinal view of the aorta; D. Simultaneous recording of intra-aortic blood pressure.

Wilcoxon signed rank test was used. $\mathrm{P}$ values of $<0.05$ were considered statistically significant.

\section{Results}

Twelve patients with evidence from noninvasive imaging (TTE and CT-angiography) for ascending aortic enlargement were included in the present study. Of these $91 \%$ were males with a mean age $67 \pm 9.5$ years, all of them had arterial hypertension, $28.6 \%$ had ischemic heart disease, $42.9 \%$ had lower extremity artery disease, $42.9 \%$ were diabetics, $28.6 \%$ were smokers. Mean IVUS maximal ascending aortic diameter was $49.8 \mathrm{~mm}$, whereas mean maximal CT-diameter $-49.2 \mathrm{~mm}$ and mean maximal TTE diameter - $47.5 \mathrm{~mm}$ (Fig. 1). Mean values of the calculated elastic properties by virtue of IVUS were as follows: compliance $0.021 \pm$ \pm 0.02 ; strain $205 \pm 4.3$; aortic stiffness index $4.3 \pm$ \pm 0.75 ; elastic modulus $0.31 \pm 0.05$. In paired T-test analysis maximum ascending aortic diameter measured by CT aortography and IVUS did not differ significantly $(t=-0.19, p=0.985)$. However, there was a significant difference between IVUS measurements and TTE derived diameters $(t=13.118$, $\mathrm{p}=0.034)$. On average, IVUS diameters were $2.3 \mathrm{~mm}$ larger than the results acquired by TTE
(95\% confidence interval: 14.21-17.13). IVUS examination of the ascending aorta provided larger diameters than the ones collected by means of TTE. However, IVUS measurements did not differ significantly from diameters derived by CT aortography.

\section{Discussion}

Large arteries in the human body are also called elastic, contain a high number of collagen and elastin filaments in their medial layer which act as an elastic buffering chamber storing nearly $50 \%$ of the left ventricular (LV) stroke volume during systole [1]. In diastole, the elastic forces of the aortic wall release this residual volume to the peripheral circulation, thus creating a nearly continuous peripheral blood flow. This phenomenon is known as the Windkessel effect [2]. This function of the elastic arteries enables maintaining a relatively constant pressure in the arteries despite intermittent LV ejection and the pulsating nature of blood flow. This interaction impacts not only the peripheral circulation but also the heart, resulting in a reduction of LV afterload and improvement in coronary blood flow and LV relaxation. As the largest artery in the human body, 


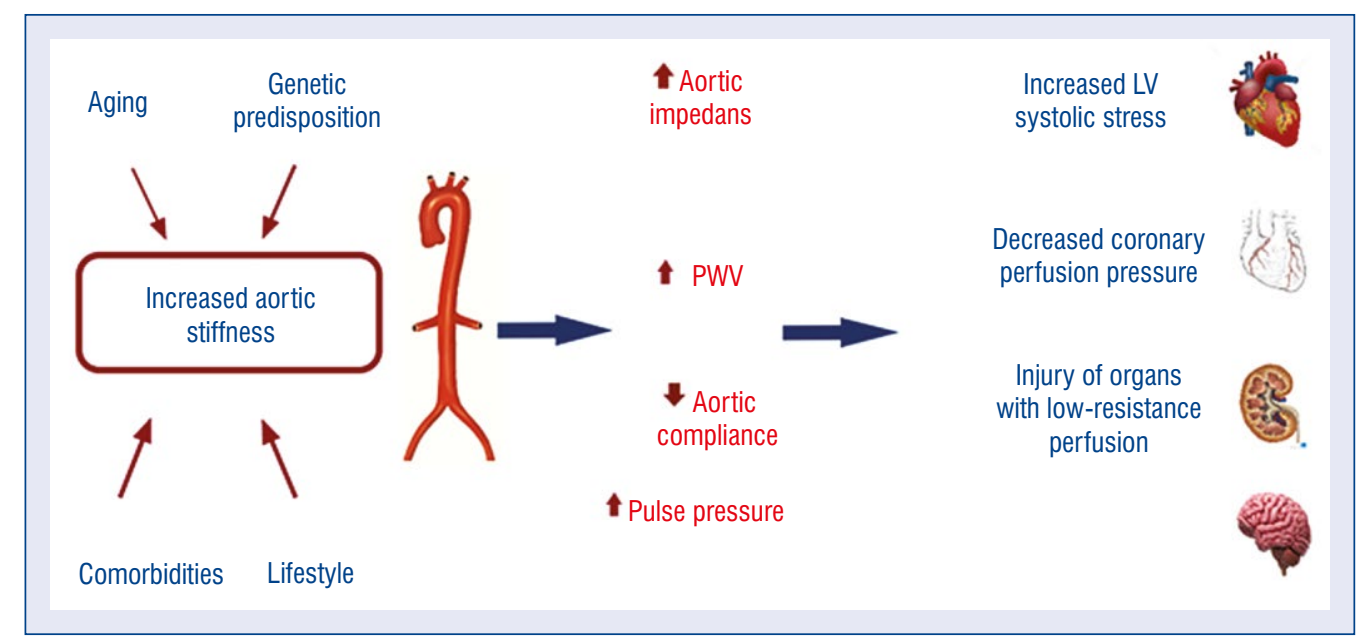

Figure 2. Pathophysiology of aortic stiffness; PWV — pulse wave velocity; LV — left ventricular.

the aorta is most prone to abnormal stiffening in response to the cumulative exposure to hemodynamic loading, lifestyle and cardiovascular (CV) risk factors. The most distensible segment of the vessel is the ascending aorta; hence it exhibits the earliest changes with aging [3]. The medial layer of the aortic wall (tunica media) is the main determinant of this process. With aging, exposure to risk factors and the development of variety of chronic diseases, the vascular wall is being progressively injured [8]. This injury provokes an inflammatory process with endothelial disfunction, elastin degradation and collagen deposition in the vessel wall. There is plenty of evidence demonstrating the relationship between diabetes, chronic kidney disease and aortic stress [9-12].

\section{Hemodynamic consequences of increased aortic stiffness}

As stated earlier a compliant aorta in a young healthy individual has the quality of effectively buffering excess pulsatility caused by the intermittent LV ejection and exhibits a slow pulse wave velocity [2]. In such conditions, reflected waves arrive to the heart during diastole, increasing diastolic coronary perfusion pressure but not systolic ventricular load. However, with a decrease of aortic distensibility and rise in aortic root impedance, there is an increase in forward wave amplitude and pulse-wave velocity (PWV). The higher PWV induces an earlier arrival of the reflected waves - in mid to late systole. This in turn, leads to systolic pressure augmentation with increased LV oxygen demand and on the other hand a decrease of coronary perfusion pressure [13]. Therefore, aortic stiffening plays central role in a vicious cycle of hemodynamic consequences, causing systolic hypertension, promoting $\mathrm{LV}$ remodeling and heart failure [14]. Importantly, aortic stiffening may be harmful farther from the heart. Transmission of pulsatile energy with excessive shear forces into the microvasculature is injuring organs in low resistance beds such as the brain and the kidney (Fig. 2) [15, 16].

\section{Aortic stress and cardio-vascular outcomes}

A large body of evidence has demonstrated the prognostic value of aortic stress for the prediction of CV events [17]. Large artery stiffness has been proved as an independent predictor of mortality as well as predictor of ischemic heart disease and stroke in the general population [4]. Furthermore, decreased arterial distensibility has been associated with increased morbidity and both all-cause and CV mortality in hypertensive patients [18]. Meta-analysis of prospective studies including one with 17,635 participants found that increased arterial stiffness is a strong predictor for coronary heart disease, stroke, and overall CV disease, respectively [5].

\section{Current methods for evaluation of arterial elastic properties}

Although, current diagnostic methods do not provide direct in vivo measurement of arterial mechanical properties, parameters can be derived by assessment of the speed at which the pressure waves propagate along the arterial tree - arterial PWV. The latter is the most validated method for noninvasive evaluation of arterial stress. Given its simplicity and accuracy for prediction of ad- 
verse CV outcomes [19, 20] it is recommended by American and European guidelines [21-23] for the management of arterial hypertension as the first-line method for assessing of arterial stiffness. PWV can be determined by measuring the pulse transit time from the pressure waveforms at two sites along a vascular segment. Carotid-femoral PWV is the current reference method for aortic stiffness and is considered to be a global estimate of arterial PWV through the entire aorta [24]. The main advantage of the method is the superficial location of the common carotid and femoral arteries. However, the acquisition of carotid and femoral pulse waveforms can be technically difficult in heavy weight patients. Another method for evaluation of large artery stiffness is the acquiring of phonocardiographic data, along with brachial and ankle cuff waveforms and the estimation of the cardiac-ankle vascular index [25]. The heart-toankle travel time is derived as the time between the pulse onset at the heart and the upstroke of the ankle waveform. A potential disadvantage is the inclusion of a long muscular arterial segment (femoral to ankle), which may distort large artery stiffness measurements. Weintraub et al. [26] in 1990 is the first to use IVUS for the assessment of a patient with aortic dissection. Since then, there are several reports and studies described in the literature about the application of IVUS for imaging of the diseased aorta [27-29]. In 1985 Hughes et al. [30] described an in vivo experiment to evaluate aortic distention in dogs by intravascular ultrasonic catheter with simultaneous measurement of intra-aortic pressure. Consequently, the canine aorta were excised and pressure/radius ratio measurements were performed once again. The authors concluded that measurement of arterial dimensions with this ultrasonic system could provide useful evaluations of the elastic properties of the aorta. Nearly, 10 years later Hansen et al. [31], performed a study with an evaluation of aortic mechanical properties in 6 patients via IVUS imaging. Minimum and maximal aortic diameters were measured, with simultaneous recording of intraaortic pressures. The authors concluded that IVUS provides an accurate in vivo evaluation of human arterial compliance, the practical value of which needs to be established.

The heterogenecity in the arterial stiffness is an important fact that should be taken into consideration when evaluating vascular elasticity properties. Every arterial segment has different viscoelastic properties, and it is inaccurate to extrapolate segmental arterial properties to the whole arterial tree. For instance, in hypertensive patients and those with other $\mathrm{CV}$ risk factors, cases have revealed the aorta stiffens earlier than other elastic arteries, such as carotid and femoral arteries. Thus, aortic and carotid stiffness cannot be used as interchangeable predictors in high-risk patients. Furthermore, with commonly used methods, measurements are conducted between two peripheral sites, whereas the most distensible segment of the aorta - the ascending part, exhibits the earliest changes with aging and is actually excluded from the analysis. The distance between measurement sites needs to be derived from surface measurements and thus represents only an approximation. What also deserves to be mentioned is the amplification phenomenon. It is well-known that there is a progressive increase in the amplitude of the pressure wave due to reflections in the distal and more muscular arteries owing to their reduced elasticity [32]. Furthermore, the stiffness of medium-sized arteries is under vasomotor regulation by the endothelial function, the sympathetic nervous system [33, $34]$ and the renin-angiotensin system [35]. Hence, blood pressure varies along the arterial tree and a method evaluating aortic wall properties locally and measuring arterial pressure simultaneously may be of specific value.

The present study is the first to assess aortic elastic properties by virtue of IVUS. Suggested herein, is that IVUS may provide accurate in-vivo measurement of aortic diameters that are acquired intravascularly and importantly, simultaneous with intra-aortic pressures recording. In the current study, IVUS provided measurements that did not differ significantly from the ones derived by the gold-standard technique - CT aortography. This encourages us to deduce that measurements devised by IVUS are accurate and that this imaging modality may be of specific value for the assessment of aortic elastic properties.

Further study evaluating a larger cohort of patients comparing IVUS with the current referent method - PWV, is needed.

\section{Conclusions}

Aortic stiffness is now recognized as an important determinant of $\mathrm{CV}$ morbidity and mortality. For years, different methods have been studied in the assessment of aortic mechanics and stress. However, none of the described methods allow simultaneous measurements of local vascular changes - pressure/diameter. In the present 
study IVUS examination of the ascending aorta provided larger diameters than the ones collected by means of TTE. However, IVUS measurements did not differ significantly from diameters derived by CT aortography

\section{Conflict of interest: None declared}

\section{References}

1. Kassab GS. Biomechanics of the cardiovascular system: the aorta as an illustratory example. J R Soc Interface. 2006; 3(11): 719 -740, doi: 10.1098/rsif.2006.0138, indexed in Pubmed: 17015300.

2. Belz GG. Elastic properties and Windkessel function of the human aorta. Cardiovasc Drugs Ther. 1995; 9(1): 73-83, doi: 10.1007/BF00877747, indexed in Pubmed: 7786838.

3. Redheuil A, Yu WC, Wu CO, et al. Reduced ascending aortic strain and distensibility: earliest manifestations of vascular aging in humans. Hypertension. 2010; 55(2): 319-326, doi: 10.1161/HYPERTENSIONAHA.109.141275, indexed in Pubmed: 20065154.

4. Mattace-Raso FUS, van der Cammen TJM, Knetsch AM, et al. Arterial stiffness and risk of coronary heart disease and stroke: the Rotterdam Study. Circulation. 2006; 113(5): 657-663, doi: 10.1161/CIRCULATIONAHA.105.555235, indexed in Pubmed: 16461838.

5. Vlachopoulos C, Aznaouridis K, Stefanadis C. Prediction of cardiovascular events and all-cause mortality with arterial stiffness: a systematic review and meta-analysis. J Am Coll Cardiol. 2010; 55(13): 1318-1327, doi: 10.1016/j.jacc.2009.10.061, indexed in Pubmed: 20338492.

6. O'Rourke MF, Staessen JA, Vlachopoulos C, et al. Clinical applications of arterial stiffness; definitions and reference values. Am J Hypertens. 2002; 15(5): 426-444, doi: 10.1016/s08957061(01)02319-6, indexed in Pubmed: 12022246.

7. Reference Values for Arterial Stiffness' Collaboration. Determinants of pulse wave velocity in healthy people and in the presence of cardiovascular risk factors: ,establishing normal and reference values'. Eur Heart J. 2010; 31(19): 2338-2350, doi: 10.1093/eurheartj/ehq165, indexed in Pubmed: 20530030.

8. Cavalcante J, Lima J, Redheuil A, et al. Aortic stiffness: current understanding and future directions. J Am Coll Cardiol. 2011; 57(14): 1511-1522, doi: 10.1016/j.jacc.2010.12.017.

9. Lee JMS, Shirodaria C, Jackson CE, et al. Multi-modal magnetic resonance imaging quantifies atherosclerosis and vascular dysfunction in patients with type 2 diabetes mellitus. Diab Vasc Dis Res. 2007; 4(1): 44-48, doi: 10.3132/dvdr.2007.005, indexed in Pubmed: 17469043.

10. Stacey RB, Bertoni AG, Eng J, et al. Modification of the effect of glycemic status on aortic distensibility by age in the multi-ethnic study of atherosclerosis. Hypertension. 2010; 55(1): 26-32, doi: 10.1161/HYPERTENSIONAHA.109.134031, indexed in Pubmed: 19933927.

11. Safar M, London G, Plante G. Arterial stiffness and kidney function. Hypertension. 2004; 43(2): 163-168, doi: 10.1161/01. hyp.0000114571.75762.b0.

12. Doyle A, Mark PB, Johnston N, et al. Aortic stiffness and diastolic flow abnormalities in end-stage renal disease assessed by magnetic resonance imaging. Nephron Clin Pract. 2008; 109(1): c1-c8, doi: 10.1159/000130170, indexed in Pubmed: 18463420.
13. Gillebert TC, Lew WY. Influence of systolic pressure profile on rate of left ventricular pressure fall. Am J Physiol. 1991; 261(3 Pt 2): H805-H813, doi: 10.1152/ajpheart.1991.261.3.H805, indexed in Pubmed: 1887926.

14. Chirinos JA, Segers P, Hughes T, et al. Large-artery stiffness in health and disease: JACC state-of-the-art review. J Am Coll Cardiol. 2019; 74(9): 1237-1263, doi: 10.1016/j.jacc.2019.07.012, indexed in Pubmed: 31466622.

15. O'Rourke MF, Safar ME. Relationship between aortic stiffening and microvascular disease in brain and kidney: cause and logic of therapy. Hypertension. 2005; 46(1): 200-204, doi: 10.1161/01. HYP.0000168052.00426.65, indexed in Pubmed: 15911742.

16. Hashimoto J, Ito S. Central pulse pressure and aortic stiffness determine renal hemodynamics: pathophysiological implication for microalbuminuria in hypertension. Hypertension. 2011; 58(5): 839-846, doi: 10.1161/HYPERTENSIONAHA.111.177469, indexed in Pubmed: 21968753.

17. Laurent S, Boutouyrie P, Asmar R, et al. Aortic stiffness is an independent predictor of all-cause and cardiovascular mortality in hypertensive patients. Hypertension. 2001; 37(5): 1236-1241, doi: 10.1161/01.hyp.37.5.1236, indexed in Pubmed: 11358934 .

18. Laurent S, Katsahian S, Fassot C, et al. Aortic stiffness is an independent predictor of fatal stroke in essential hypertension. Stroke. 2003; 34(5): 1203-1206, doi: 10.1161/01. STR.0000065428.03209.64, indexed in Pubmed: 12677025.

19. Ben-Shlomo Y, Spears M, Boustred C, et al. Aortic pulse wave velocity improves cardiovascular event prediction: an individual participant meta-analysis of prospective observational data from 17,635 subjects. J Am Coll Cardiol. 2014; 63(7): 636-646, doi: 10.1016/j.jacc.2013.09.063, indexed in Pubmed: 24239664.

20. Asmar RG, Topouchian JA, Benetos A, et al. Non-invasive evaluation of arterial abnormalities in hypertensive patients. J Hypertens Suppl. 1997; 15(2): S99-107, doi: 10.1097/00004872199715022-00010, indexed in Pubmed: 9218206.

21. Townsend RR, Wilkinson IB, Schiffrin EL, et al. Recommendations for Improving and Standardizing Vascular Research on Arterial Stiffness: A Scientific Statement From the American Heart Association. Hypertension. 2015; 66(3): 698-722, doi: 10.1161/HYP.0000000000000033, indexed in Pubmed: 26160955.

22. Lehmann ED, Parker JR, Hopkins KD, et al. Validation and reproducibility of pressure-corrected aortic distensibility measurements using pulse-wave-velocity Doppler ultrasound. J Biomed Eng. 1993; 15(3): 221-228, doi: 10.1016/0141-5425(93)90118-i, indexed in Pubmed: 8320981.

23. Williams B, et al. $2018 \mathrm{ESC} / \mathrm{ESH}$ Guidelines for the management of arterial hypertension: The Task Force for the management of arterial hypertension of the European Society of Cardiology (ESC) and the European Society of Hypertension (ESH). Eur Heart J. 2018; 39(33): 3021-3104.

24. Laurent S, Cockcroft J, Van Bortel L, et al. Expert consensus document on arterial stiffness: methodological issues and clinical applications. Eur Heart J. 2006; 27(21): 2588-2605, doi: 10.1093/ eurheartj/ehl254, indexed in Pubmed: 17000623.

25. Sugawara J, Hayashi K, Yokoi T, et al. Brachial-ankle pulse wave velocity: an index of central arterial stiffness? J Hum Hypertens. 2005; 19(5): 401-406, doi: 10.1038/sj.jhh.1001838, indexed in Pubmed: 15729378.

26. Weintraub AR, Schwartz SL, Pandian NG, et al. Evaluation of acute aortic dissection by intravascular ultrasonography. N Engl J Med. 1990; 323(22): 1566-1567, doi: 10.1056/ NEJM199011293232215, indexed in Pubmed: 2233942. 
27. Alfonso F, Goicolea J, Aragoncillo P, et al. Diagnosis of aortic intramural hematoma by intravascular ultrasound imaging. Am J Cardiol. 1995; 76(10): 735-738, doi: 10.1016/s00029149(99)80213-6, indexed in Pubmed: 7572641.

28. Mileva N, Vassilev D, Gil R, et al. Misdiagnosed aortic intramural hematoma and the role of intravascular ultrasound imaging in detection of acute aortic syndrome: a case report. Cardiovasc Innov Appl. 2018; 2(4): 447-449, doi: 10.15212/cvia.2017.0028.

29. Wei Hu, Schiele F, Meneveau N, et al. The value of intravascular ultrasound imaging in diagnosis of aortic penetrating atherosclerotic ulcer. EuroIntervention. 2006; 1(4): 432-437, indexed in Pubmed: 19755218.

30. Hughes DJ, Fearnot NE, Babbs CF, et al. Continuous measurement of aortic radius change in vivo with an intra-aortic ultrasonic catheter. Med Biol Eng Comput. 1985; 23(3): 197-202, doi: 10.1007/BF02446857, indexed in Pubmed: 4021563.

31. Hansen ME, Yucel EK, Megerman J, et al. In vivo determination of human arterial compliance: preliminary investigation of a new technique. Cardiovasc Intervent Radiol. 1994; 17(1): 22-26, doi: 10.1007/BF01102067, indexed in Pubmed: 8187128.

32. Latham RD, Westerhof N, Sipkema P, et al. Regional wave travel and reflections along the human aorta: a study with six simultaneous micromanometric pressures. Circulation. 1985; 72(6): 1257-1269, doi: 10.1161/01.cir.72.6.1257, indexed in Pubmed: 4064270.

33. Boutouyrie P, Lacolley P, Girerd X, et al. Sympathetic activation decreases radial artery compliance in humans. Am J Physiol. 1994; 267: H1368-H1376.

34. Giannattasio C, Failla M, Lucchina S, et al. Arterial stiffening influence of sympathetic nerve activity: evidence from hand transplantation in humans. Hypertension. 2005; 45(4): 608-611, doi: 10.1161/01.HYP.0000157368.09939.88, indexed in Pubmed: 15699439.

35. Giannattasio C, Failla M, Stella ML, et al. Angiotensin-converting enzyme inhibition and radial artery compliance in patients with congestive heart failure. Hypertension. 1995; 26(3): 491-496, doi: 10.1161/01.hyp.26.3.491, indexed in Pubmed: 7649587. 